\title{
Social deficiencies in the tourist municipalities of the riviera maya in the state of Quintana Roo, Mexico
}

\section{Carencias sociales en los municipios turísticos de la riviera maya en el estado de Quintana Roo, México}

DOI: $10.46932 / \mathrm{sjjdv3n1-123}$

Received in: January $20^{\text {th }}, 2022$

Accepted in: February $1^{\text {st }}, 2022$

\section{Carlos Alberto Pérez Canul}

Doctor en Ciencias de la Administración

Institución: Universidad Autónoma de Campeche

Dirección: Av. Agustín melgar s/n, Col. Buenavista, Campeche, México

Correo electrónico: caaperez@uacam.mx

\section{Charlotte Monserrat Llanes Chiquini}

Doctor en Ciencias de la Administración

Institución: Universidad Autónoma de Campeche

Dirección: Av. Agustín melgar s/n, Col. Buenavista, Campeche, México

Correo electrónico: chmllane@uacam.mx

\section{Roger Manuel Patrón Cortés}

Doctor en Ciencias de la Administración

Institución: Universidad Autónoma de Campeche

Dirección: Av. Agustín melgar s/n, Col. Buenavista, Campeche, México

Correo electrónico: Roger_patron_cortes@hotmail.com

Thania del Carmen Tuyub Ovalle

Maestra en Ciencias de la Educación

Institución: Universidad Autónoma de Campeche

Dirección: Av. Agustín melgar s/n, Col. Buenavista, Campeche, México

Correo electrónico: thctuyub@uacam.mx

\section{RESUMEN}

El estado de Quintana Roo con la fundación de la ciudad de Cancún en 1970, se convirtió en el principal sitio turístico de México. Esta situación origino que se desarrollara el corredor turístico conocido como Riviera Maya y que actualmente es uno de los principales polos turísticos del país. Ciudades como Playa del Carmen y Tulum han tenido un desarrollo importante y actualmente son lugares con un gran impacto para la industria turística. Es importante destacar que la región ha tenido un desarrollo económico en los últimos 50 años, pero diversas regiones del país no se desarrollaron de la misma manera, la mayoría de los estudios sobre el desarrollo de México muestran que el país ha sido regionalmente desequilibrado y la tendencia apunta a un agravamiento de esos desequilibrios en el futuro, por lo que frente a este panorama son los gobiernos estatales y los actores de la sociedad civil los que deben asumir una posición de liderazgo proactivo que permita combatir los problemas sociales. En una primera etapa es importante partir de un análisis claro de la situación de la pobreza en cada entidad federativa analizando las diversas variables que influyen en esta situación. Este documento presenta los resultados de la investigación "Carencias sociales en los municipios turísticos de la Riviera Maya en el estado de Quintana Roo, México". El cual propone un análisis de diversas variables que influyen en la medición de la pobreza, mediante el índice 
de Rezago Social (IRS) diseñado por el Consejo Nacional de Evaluación de la Política de Desarrollo Social (CONEVAL) para determinar las carencias sociales existentes en cada municipio de la región de la Riviera Maya del estado de Quintana Roo, México

Palabras clave: rezago social, calidad de vida, desarrollo social.

\begin{abstract}
The State of Quintana Roo with the foundation of the city of Cancun in 1970, became the main tourist attraction in Mexico. This situation led to the development of the tourist corridor known as the Riviera Maya, which is currently one of the most important tourist sites in the country. Cities like Playa del Carmen and Tulum have undergone significant development and are currently places with a great impact on the tourism industry. It is important to note that the region has had an economic development in the last 50 years, but various regions of the country did not develop in the same way, most studies on the development of Mexico show that the country has been regionally unbalanced and the trend points to a worsening of these imbalances in the future, so that, faced with this panorama, it is the state governments and the actors of civil society that must assume a position of proactive leadership that allows them to combat social problems. First of all, it is important to start with a clear analysis of the poverty situation in each federal entity, analyzing the several variables that influence this situation. This document presents the results of the research "Social deprivation in the tourist municipalities of the Riviera Maya in the state of Quintana Roo, Mexico". Which proposes an analysis of various variables that influence the measurement of poverty, through the Social Lag Index (IRS) designed by the National Council for the Evaluation of Social Development Policy (CONEVAL) to determine the existing social deficiencies in each municipality in the Riviera Maya region of the state of Quintana Roo, Mexico
\end{abstract}

Keywords: social backwardness, quality of life, social development.

\title{
1 INTRODUCCIÓN
}

En lo que se refiere a la medición de la pobreza, en cumplimiento de las disposiciones establecidas en la Ley General de Desarrollo Social (LGDS), el Consejo Nacional de Evaluación de la Política de Desarrollo Social (CONEVAL) tiene la responsabilidad de informar las estimaciones de pobreza en México y para cada entidad federativa. La finalidad de esta información es proporcionar elementos para mejorar las políticas públicas tendientes a la superación de la pobreza en México. Al conocer el estado que guardan las dimensiones que conforman el fenómeno de la pobreza en México los órdenes de gobierno (federal, estatal y municipal) pueden identificar las áreas en las que se requiere redoblar los esfuerzos institucionales, así como las regiones del país donde es necesario fortalecer la atención prioritaria de la población en situación de pobreza o vulnerabilidad económica o social. ( Consejo Nacional de Evaluación de la Política de Desarrollo Social, 2014)

\section{OBJETIVOS DEL ESTUDIO}

El objetivo general de la investigación consiste en medir la carencia social de los habitantes de los municipios de la región de la Riviera Maya en el estado de Quinta Roo, México con respecto al Índice de 
Rezago Social (IRS) del Consejo Nacional de Evaluación de la Política de Desarrollo Social (CONEVAL).

\section{MARCO TEÓRICO}

\subsection{CONCEPTUALIZACIÓN DE LA POBREZA}

Para conceptualizar la pobreza se requiere definir quiénes son los pobres, se considera que primero se deben cuantificar los entes, característica o situación, para ello es imprescindible tener claro el concepto a medir, por lo que es necesario elegir el concepto de pobreza, pues ello condicionará el enfoque con el que se trabajará. El cuantificar la pobreza implica encontrarse entre las nociones de pobreza "absoluta" y "relativa", entre los enfoques "directo" e "indirecto" y entre las perspectivas "objetiva" y "subjetiva" (Ravallion , 2003). Vale la pena recordar que ningún método de identificación y agregación es por sí solo suficiente, por lo que el uso combinado de los mismos puede ser una opción más acertada para la cuantificación de la pobreza. (Ortiz \& Ríos, 2013)

Una medida primaria de la pobreza es unidimensional, la cual consiste sencillamente en partir del umbral de pobreza y todas las personas que se encuentran debajo del umbral son pobres, con ello se obtiene una tasa de pobreza, la cual consiste en dividir el número de pobres en la sociedad, entre la población total. Generalmente se considera que esta medida es deficiente, pues no indica la proporción en que el ingreso de las personas está por debajo del umbral ni qué carencia es la más arraigada.

La pobreza es un fenómeno multidimensional que afecta la vida de las personas, niega la igualdad de oportunidades y las excluye de las actividades económicas, sociales y culturales de su comunidad. La pobreza se refleja no sólo en bajos niveles de ingreso, sino también en las precarias condiciones de vida de la población, es decir, en la imposibilidad de acceder a una alimentación adecuada, a servicios educativos, a servicios de salud, a fuentes formales de empleo, a una vivienda digna, a una pensión, entre otros aspectos. Esta situación puede propiciar que las familias y los individuos experimenten por varias generaciones condiciones de pobreza (Consejo Nacional de Evaluación de la Política de Desarrollo Social, 2014).

Para el CONEVAL (2014) la pobreza multidimensional implica que una persona se encuentra en situación de pobreza multidimensional cuando no tiene garantizado el ejercicio de al menos uno de sus derechos para el desarrollo social, y si sus ingresos son insuficientes para adquirir los bienes y servicios que requiere para satisfacer sus necesidades. (Ortiz \& Ríos, 2013)

Para establecer una medida multidimensional de pobreza es necesario: i) definir el marco conceptual adoptado para su medición; ii) precisar la unidad de observación del estudio (individuo u hogar); iii) delimitar las dimensiones así como las variables e indicadores que las caracterizan; iv) 
establecer la importancia relativa de cada dimensión y, en consonancia, estipular los ponderadores de los indicadores; v) seleccionar los umbrales de pobreza (satisfacción mínima) tanto para cada dimensión como de manera global para la identificación de las personas que la padecen, es decir, quién es pobre según el punto de vista multidimensional, y vi) especificar los métodos de agregación tanto dentro como por medio de las dimensiones. Además, y no menos importante, es necesario contar con la fuente de información que permita medir cada una de las dimensiones propuestas según el enfoque teórico adoptado ( Consejo Nacional de Evaluación de la Política de Desarrollo Social, 2014)

\subsection{DEFINICIÓN, IDENTIFICACIÓN Y MEDICIÓN DE LA POBREZA EN MÉXICO}

En México, hasta antes de la promulgación de la Ley General de Desarrollo Social (LGDS) en 2004 y la creación del Consejo Nacional de Evaluación de la Política de Desarrollo Social (CONEVAL) mandatado por ésta, la mayoría de los estudios sobre medición y análisis de la pobreza estaba basada en una perspectiva unidimensional, la cual utilizaba el ingreso como una aproximación del bienestar económico de la población. Con esta perspectiva, la identificación de la población en pobreza se realiza de manera indirecta, es decir, se contrasta el ingreso de las personas con una línea de pobreza que permite valorar si dicho ingreso es insuficiente para satisfacer sus necesidades y, por ende, determinar si la persona es pobre o no. ( Consejo Nacional de Evaluación de la Política de Desarrollo Social, 2014)

El concepto y las mediciones de la pobreza han estado sujetos a una gran polémica, particularmente entre las visiones opuestas del enfoque biológico o de mera subsistencia y el de privación o pobreza relativa. Adoptar uno u otro tiene una enorme repercusión en los cálculos sobre la incidencia e intensidad de la pobreza e incluso sobre el signo de su evolución a lo largo del tiempo. (Boltvinik, 1997)

En las metrópolis modernas han surgido nuevas categorías de pobres cuyos niveles de carencias son menores a los definidos para el medio rural, pero son reveladoras de nuevos mecanismos de empobrecimiento, desventaja y desigualdad que impactan, de manera directa, en la situación económica, cultural, social e institucional de las ciudades y dejan al margen de las ventajas de la modernidad a aquella población que ahora se identifica como nuevos pobres (Padilla, 2011).

Bajo el entendido de que la pobreza es un fenómeno multidimensional cuyas causas centrales van más allá del ingreso y se relacionan con carencias vitales que los pobres enfrentan, entre las más recurrentes están las relacionadas con la falta de acceso al empleo formal, a la educación y servicios de salud; la vulnerabilidad que presenta ante situaciones de crisis; la exclusión y la falta de voz y participación social, por lo que la metodología de análisis deberá incluir en enfoques tanto cuantitativo como cualitativo. En este contexto, la ciudad de México, como capital del país y cuya primacía se ha mantenido desde principios del siglo XX hasta nuestros días, su población ha sido testigo del deterioro de 
las condiciones de vida en una urbe cuya capacidad de generar empleo de calidad, bien remunerado, es cada vez más limitada para una amplia mayoría; esta coyuntura favorece la desigualdad social y la presencia de los nuevos pobres urbanos quienes han sido poco estudiados debido a que el interés del Estado se ha enfocado, principalmente, al medio rural (Padilla, 2011).

Debido al desarrollo económico de la región en las últimas décadas. Debemos mencionar la contribución de Sobrino al conocimiento sobre la naturaleza competitiva de las ciudades en México (Sobrino 2005), La competitividad no es un atributo que puede ser medido directamente, sino se debe adoptar algún indicador de la misma; en este caso se eligió el crecimiento del valor bruto de la producción (VBP) industrial, comercial y de servicios. La justificación de estos sectores como indicadores de competitividad económica local es la siguiente: el crecimiento en las ventas manufactureras refleja inversiones que se traducen en ampliación de su base exportadora y generación de empleo; el crecimiento de los ingresos derivados del comercio y servicios reflejan el dinamismo demográfico de la ciudad, incremento en el ingreso de los habitantes y atractividad de no residentes para ir de compras, acceder a servicios de educación y salud o por motivos turísticos recreativos y culturales. (Sobrino, 2005)

Para poner en operación la propuesta anterior de ventajas competitivas locales y explorar el desempeño competitivo de las ciudades del estudio, se construyó una base de datos con los siguientes indicadores cuantitativos: Ventajas competitivas empresariales: i) Productividad parcial del trabajo; ii) Remuneración promedio al personal ocupado (sueldos); iii) Intensidad del capital, o relación capitaltrabajo (capital); iv) Tamaño promedio de los establecimientos (tamaño), y v) Porcentaje del personal ocupado en la industria maquiladora de exportación respecto a la demanda ocupacional de la estructura productiva local. Las tres primeras estiman la eficiencia microeconómica de los establecimientos manufactureros (Ballance 1987; González y Mariña 1992; Roper 1998) y la cuarta es una acotación de la concentración técnica. (Utton, 1975)

\section{METODOLOGÍA}

Enfoque.- El estudio presenta un enfoque cuantitativo basado en mediciones numéricas y análisis estadístico que establecen patrones de comportamiento.

Tipo.-El estudio es descriptivo, ya que únicamente pretende medir información de manera conjunta sobre las variables de referencia.

\section{A. Participantes en el estudio}

Universo y muestra. - Los participantes de estudio fueron la totalidad de los municipios en el estado de Quinta Roo, excepto el municipio de Puerto Morelos que fue constituido en noviembre de 2015. 
Los diez municipios son: Municipio de Othón P. Blanco, en donde se asienta Chetumal la capital del Estado y la cabecera municipal; Municipio de Felipe Carrillo Puerto, cuya cabecera municipal es la ciudad del mismo nombre; Municipio de José María Morelos con cabecera municipal en la ciudad de José María Morelos; Municipio de Solidaridad, cabecera municipal Playa del Carmen; Municipio de Cozumel cuya cabecera municipal es la ciudad de Cozumel; Municipio de Isla Mujeres, cabecera municipal la ciudad de Isla Mujeres; Municipio de Benito Juárez, cabecera municipal la ciudad de Cancún; el Municipio de Lázaro Cárdenas cuya cabecera municipal es Kantunilkín ; el Municipio de Tulúm, cuya cabecera municipal es el poblado de Tulúm y el último municipio es el de Bacalar.

Instrumento. La metodología y el instrumento fue desarrollado por el CONEVAL para la medición del índice de rezago social (IRS).

Metodología. En el documento Los Mapas de Pobreza en México, junto con su anexo estadístico y geográfico, se dan a conocer los resultados de las estimaciones realizadas por el CONEVAL para la medición multidimensional de la pobreza a nivel estatal y municipal. Los mapas muestran dos medidas de carencias: la pobreza por ingresos y el índice de rezago social. Estas mediciones se construyeron a partir de los indicadores que marca la Ley General de Desarrollo Social y que deben ser considerados en la definición, identificación y medición de la pobreza.

\section{El Índice de Rezago Social}

La construcción del índice de rezago social tiene como intención cumplir con tres criterios básicos:

En primer lugar, se consideró la oportunidad de la información, incorporando la información disponible acorde a los indicadores de pobreza y a los niveles de desagregación que marca la Ley y de la información disponible.

En segundo lugar, se seleccionó una base de datos cuya estructura permitiera obtener indicadores en los niveles de agregación de localidades, municipal, estatal y nacional. Sobre la base de estos dos primeros criterios se decidió utilizar la base de datos

En tercer lugar, se optó por la técnica estadística de componentes principales ya que permite resumir en un indicador agregado las diferentes dimensiones del fenómeno en estudio.

Para la construcción del índice de rezago social se consideraron los siguientes indicadores:

- Educativos.

- Acceso a servicios de salud.

- Calidad y espacios en la vivienda.

- Servicios básicos en la vivienda.

- Activos en el hogar. 
El índice resultante permite ordenar las unidades de observación (localidad, municipio, estado) según sus carencias sociales. Además, esta técnica es la misma que utiliza CONAPO para la construcción del índice de marginación El Índice de Rezago Social se construye como una suma ponderada de los diferentes indicadores. Para su construcción se utilizan como ponderadores los coeficientes de la primera componente. El índice es estandarizado de tal forma que su media sea cero y su varianza unitaria.

De esta forma se crearon cinco estratos que son: muy bajo, bajo, medio, alto y muy alto rezago social, y que dan la idea de grupos de localidades, municipios y estados que van de aquellos que muestran un menor a una mayor carencia en los indicadores que conforman el índice, respectivamente. ( Consejo Nacional de Evaluación de la Política de Desarrollo Social, 2014)

\section{RESULTADOS}

De acuerdo con la metodología establecida para la medición del rezago social (IRS) del Consejo Nacional de Evaluación de la Política de Desarrollo Social (CONEVAL), se presentan los siguientes resultados:

Tabla 1 Resultado del índice de rezago social (IRS) para los municipios de la región de la Riviera Maya en el estado de Quinta Roo.2015.

\begin{tabular}{|c|r|r|}
\hline MUNICIPIO & IRS & $\begin{array}{c}\text { GRADO DE REZAGO } \\
\text { SOCIAL }\end{array}$ \\
\hline Cozumel & -1.133519 & Muy bajo \\
\hline Felipe Carrillo Puerto & -0.014021 & Bajo \\
\hline Isla Mujeres & -0.699807 & Bajo \\
\hline Othón P. Blanco & -0.972730 & Muy bajo \\
\hline Benito Juárez & -1.138995 & Muy bajo \\
\hline José María Morelos & 0.098653 & Bajo \\
\hline Lázaro Cárdenas & -0.216774 & Bajo \\
\hline Solidaridad & -0.989263 & Muy bajo \\
\hline Tulum & -0.393604 & Bajo \\
\hline Bacalar & -0.123589 & Bajo \\
\hline
\end{tabular}

Como se observa en la tabla 1, los municipios están ordenados de acuerdo con la clave de municipio del INEGI, sin embargo, los municipios de Lázaro Cárdenas y José María Morelos no colindan con el Mar Caribe, por lo que no están en la región de la Riviera Maya. En la tabla podemos visualizar según los resultados obtenidos por el CONEVAL para el estado de Quintana Roo fueron diferentes a los esperados, ya que en donde se observa un menor rezago social no es en el municipio de Othón P. Blanco donde está ubicada la capital del estado, , si no que el estudio muestra que el menor rezago social es en el municipio de Benito Juárez donde se encuentra la ciudad turística de Cancún y en un segundo lugar el municipio de Cozumel. 
La tabla 1 nos indica los resultados del Índice de Rezago Social (IRS), sin embargo, cabe señalar los cinco estratos que son: muy bajo, bajo, medio, alto y muy alto rezago social, y que dan la idea de grupos de localidades, municipios y estados que van de aquellos que muestran un menor a una mayor carencia en los indicadores que conforman el índice, es decir que menores carencias son negativos y en dicha escala si se tiene mayores carencias más aumentaran los números positivos en la escala.

\section{CONCLUSIONES}

Los resultados obtenidos en la medición del IRS confirman el grado de rezago social existente en los municipios de la Riviera Maya del estado de Quintana Roo, con lo que surge la necesidad de proponer e implementar políticas públicas que permitan incrementar la competitividad en la región y por ende incrementar el nivel de vida de la población, establecer una serie congruente de políticas públicas contra la pobreza que apoyaría una mejoría tanto en el nivel de vida como en la competitividad, sobre todo en los municipios en donde el índice de rezago social nos demuestra las carencias de los habitantes. 


\section{REFERENCIAS}

Boltvinik, J. (1997). Diversas visiones sobre la pobreza en México. Factores determinantes. Política y cultura, 115-135.

Ortiz, G. J., \& Ríos, B. H. (2013). La Pobreza en México, un análisis con enfoque multidimensional. Análisis Económico, XXVIII(69), 189-218.

Padilla, I. V. (2011). Pobreza y segregación territorial en la ciudad de México. Revista Geográfica de América Central, 1-14.

Ramírez, B. (1992). Modernización y restructuración territorial. Ciudades, 3-9.

Sobrino, J. (2005). Competitividad Territorial. Ámbitos e indicadores de análisis. Economia, Sociedad y Territorio, 123-183.

Social, C. N. (2014). MEDICIÓN MULTIDIMENSIONALDE LA POBREZA EN MÉXICO. El Trimestre Económico, 5-42.

Utton, M. (1975). La concentración Industrial. Madrid: Alianza. 